\title{
Reconstruction of the bronchus and pulmonary artery
}

\author{
Giulio Maurizi ${ }^{1}$, Antonio D'Andrilli ${ }^{1}$, Federico Venuta ${ }^{2,3}$, Erino Angelo Rendina ${ }^{1,3}$ \\ ${ }^{1}$ Department of Thoracic Surgery, Sant'Andrea Hospital, Sapienza University of Rome, Rome, Italy; ${ }^{2}$ Department of Thoracic Surgery, Policlinico \\ Umberto I, Sapienza University of Rome, Rome, Italy; ${ }^{3}$ Lorillard Spencer Cenci Foundation, Rome, Italy \\ Contributions: (I) Conception and design: G Maurizi; (II) Administrative support: None; (III) Provision of study materials or patients: G Maurizi; (IV) \\ Collection and assembly of data: G Maurizi; (V) Data analysis and interpretation: All authors; (VI) Manuscript writing: G Maurizi, A D’Andrilli; (VII) \\ Final approval of manuscript: All authors. \\ Correspondence to: Giulio Maurizi, MD. Department of Thoracic Surgery, Sant'Andrea Hospital, Sapienza University of Rome, Via di Grottarossa, \\ 1035, 00189 Rome, Italy. Email: giulio.maurizi@uniroma1.it.
}

\begin{abstract}
Bronchovascular reconstructive procedures employed in order to avoid pneumonectomy (PN) in patients functionally unsuitable have provided, over time, excellent results, similar or even better than those obtained by PN. In recent years, new successful techniques have been developed that pertain in particular the prevention of major complications and the reconstruction of the pulmonary artery (PA). Encouraging data from increasing number of published experiences support the choice of parenchymal sparing procedures for lung cancer also in patients with good functional reserve. This is even more true if considering trials published in the last 10 years, thus indicating that improved outcome can be achieved with increased experience in reconstructive techniques and perioperative management. This article discusses the main technical aspects and results of literature.
\end{abstract}

Keywords: Sleeve lobectomy (SL); pulmonary artery reconstruction; non-small-cell lung cancer

Submitted Oct 31, 2015. Accepted for publication Dec 18, 2015.

doi: 10.3978/j.issn.2072-1439.2016.02.06

View this article at: http://dx.doi.org/10.3978/j.issn.2072-1439.2016.02.06

\section{Introduction}

Bronchial and vascular sleeve resections have proved to be valid options for the treatment of centrally located lung cancer (1-4). After induction therapy reconstructive procedures may be indicated also when indissociable fibrotic tissue embeds the bronchus and/or the pulmonary artery (PA).

These surgical procedures are employed in order to avoid pneumonectomy $(\mathrm{PN})$ in patients with compromised cardiac and/or pulmonary function.

Although bronchovascular reconstructions associated with lobectomy are surgical procedures with higher technical difficulty if compared with standard major lung resections, postoperative morbidity and mortality data from a number of recent studies report overall better results for patients undergoing sleeve lobectomy (SL) with respect to $\mathrm{PN}$. This is even more true considering trials published in the last 10 years, thus indicating that improved outcome can be achieved with increased experience in reconstructive techniques (5-18) (Table 1). These results support the increasing choice of parenchymal sparing procedures for lung cancer also in patients with good functional reserve.

In recent years, new successful techniques have been developed $(19,20)$ that pertain in particular the prevention of major complications and the reconstruction of the PA; these provide good short- and long-term results.

Moreover, many surgeons believe that PN, particularly on the right side, is a disease itself, causing severe postoperative impairment of lung function, cardiac function, and quality of life, and therefore PN should be performed only when necessary to obtain full oncological radicality.

Since the first sleeve resections were reported in the early 1950s $(21,22)$ significant technical advances and increasing experience over time have allowed achievement of excellent clinical and oncologic results, resulting in wide utilization and consensus in the use of parenchymal sparing procedures for lung cancer. 
Table 1 Results of main series comparing SL with PN published in the last 10 years: morbidity, mortality, and long-term survival

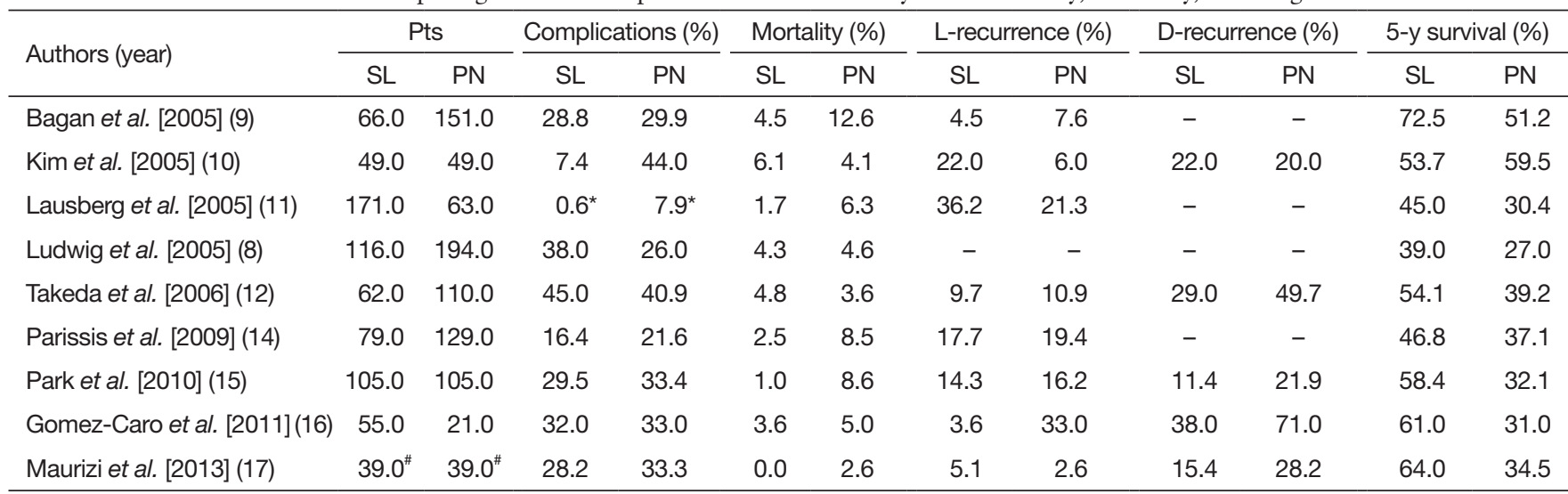

*, bronchial complications; ", all post-neoadjuvant therapy patients. L-recurrence, local recurrence; D-recurrence, distant recurrence; SL, sleeve lobectomy; PN, pneumonectomy.
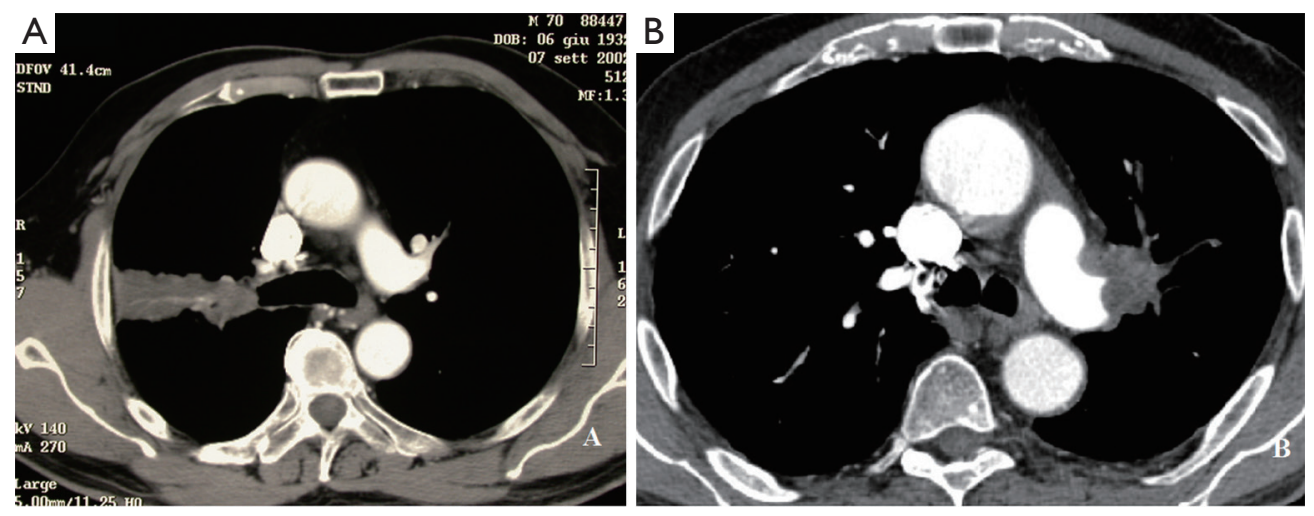

Figure 1 Indication for sleeve lobectomy (SL); computed tomography (CT) scan images. (A) The tumor arises at the origin of the right upper lobar bronchus; (B) the tumor infiltrates both the bronchus and the pulmonary artery (PA) on the left side.

\section{Bronchial resections}

Bronchial sleeve for lung cancer is indicated when a tumor infiltrates the origin of a lobar bronchus, but not to the extent that a PN is required. In addition, SL may be indicated for $\mathrm{N} 1$ lymph node infiltration of the bronchus and/or the PA, as is often the case in left upper lobe tumors that require combined broncho-vascular reconstruction. Reconstructive procedures also may be indicated after induction therapy when unremovable fibrotic tissue is embedded in the bronchus (Figure 1).

\section{Operative technique}

Bronchial sleeve resections and reconstructions are commonly performed through the same thoracotomy made for standard pulmonary resections (posterolateral or lateral muscle-sparing incisions, which are both suitable for exposure and dissection) and more recently VATS approaches have been described $(23,24)$. Our technique starts with the dissection beginning in the anterior hilum and then continuing to complete dissection of the main PA. In cases where bulky disease causes increased difficulty during dissection, the pericardium can be opened on either side in order to gain improved proximal control. Next, the main PA is surrounded with surgical umbilical tape. The subsequent steps are specific to the type of sleeve resection performed and each is described independently in the following sections. 


\section{Upper SL}

On the right side, the dissection starts superiorly at the level of the upper lobe bronchus. The lung is retracted anteriorly, and dissection is continued in the bifurcation between the upper lobe bronchus and the intermediate bronchus. The lymph node frequently found in this location, is elevated away from the bifurcation in order to expose the pulmonary arterial branch to the posterior segment of the lower lobe. Once this branch is identified, the posterior portion of the fissure is completed with a linear stapler. This approach avoids extensive parenchymal dissection in the fissure. The intermediate bronchus is encircled just distal to the right upper lobe take-off, and surgical umbilical tape is placed to aid airway division at the appropriate site. Once complete resectability is confirmed, ligation and division of the pulmonary arterial branches to the upper lobe are performed. Similarly, the pulmonary vein (PV) branch draining the upper lobe is divided with a vascular stapler, taking care to preserve the middle lobe venous drainage. The minor fissure is completed with a linear stapler. The main stem bronchus is encircled with umbilical tape at its origin.

The bronchial resection phase is then started. The main stem bronchus is divided just proximal to the upper lobe take-off. Once the bronchus has been opened, the decision to proceed with a sleeve resection may be made based on macroscopic or microscopic findings. Subsequently, the intermediate bronchus is divided just distal to the right upper lobe take-off. These cuts must be perpendicular to the long axis of the airway.

On the left side, proximal arterial control is obtained taking care to avoid injury to the short apical-posterior segmental branch of the left PA. Dissection is continued along the plane of the artery and the superior segmental branch to the lower lobe is identified. The posterior fissure is then completed with a linear stapler. The arterial segmental branches to the upper lobe are ligated and divided. The upper PV is divided with a vascular stapler. The anterior portion of the fissure is completed with a linear stapler. The main stem bronchus is encircled proximal to the bifurcation, and umbilical tape is placed. The main stem bronchus is divided proximal to the bifurcation, and the left lower lobe bronchus is divided at its origin. The origin of the superior segmental bronchus can be quite close to the origin of the lower lobe bronchus, and the lobar division must leave the bronchus intact without separating the superior segmental bronchus.

A frozen-section examination of the bronchial resection margins is performed in order to confirm the radicality of resection. Microscopic tumors found at the bronchial margin require either additional resection of the involved area or PN.

Different techniques have been described for bronchial anastomotic reconstruction. We favor the use of interrupted sutures of $4 / 0$ monofilament absorbable material $(25,26)$. The employment of continuous running suture (complete or partial) has been described by others $(16,27)$. In our technique, the first suture is placed in an "outside-to-inside" fashion at the junction of the cartilaginous and membranous bronchi. These initial sutures can be immediately tied in order to confer improved stability to this point of the anastomosis. Additional sutures are placed at $2 \mathrm{~mm}$ intervals to complete the first half of the cartilaginous anastomosis. Once the midpoint of the cartilaginous bronchus is reached, the anastomosis is then completed on the opposite side of the bronchial circumference in a similar fashion. Sutures are then tied, starting from either end of the cartilaginous portion and working toward the middle. Placing and tying the sutures in this order allows compensation for even large caliber discrepancies. This technique prevents torsion of the bronchial axis and gently stretches and dilates the circumference of the distal bronchus. The anastomosis is then tested with $20 \mathrm{~cm}$ water inflation pressure by filling the pleural cavity with saline solution. Needle hole air leaks are usually ignored; however, air leaks between the cut edges of the bronchus, if small, are reinforced with simple interrupted sutures. A large area of leakage may require replacement of the entire anastomosis. Protection of the bronchial anastomosis with a viable tissue flap is recommended by most authors $(28,29)$. The present authors routinely use an intercostal muscle flap (27), which has excellent vascularization provided by the intercostal artery, enables preservation of airtightness even in the event of small anastomotic dehiscence, and minimizes the risk of PA erosion, particularly when an associated vascular reconstruction is performed. Alternatively, the mediastinal fat pad (28), pericardial tissue or pleural tissue (27) has been used as viable flaps. For the final success of bronchial reconstruction, it is essential to avoid tension on the anastomosis. This can be achieved by dividing the pulmonary ligament or, more often on the right side, by opening the pericardium around the PV.

\section{Lower SL ("Y" sleeve)}

On the right side sleeve bilobectomy is performed for an endoluminal lesion in the bronchus intermedius that 

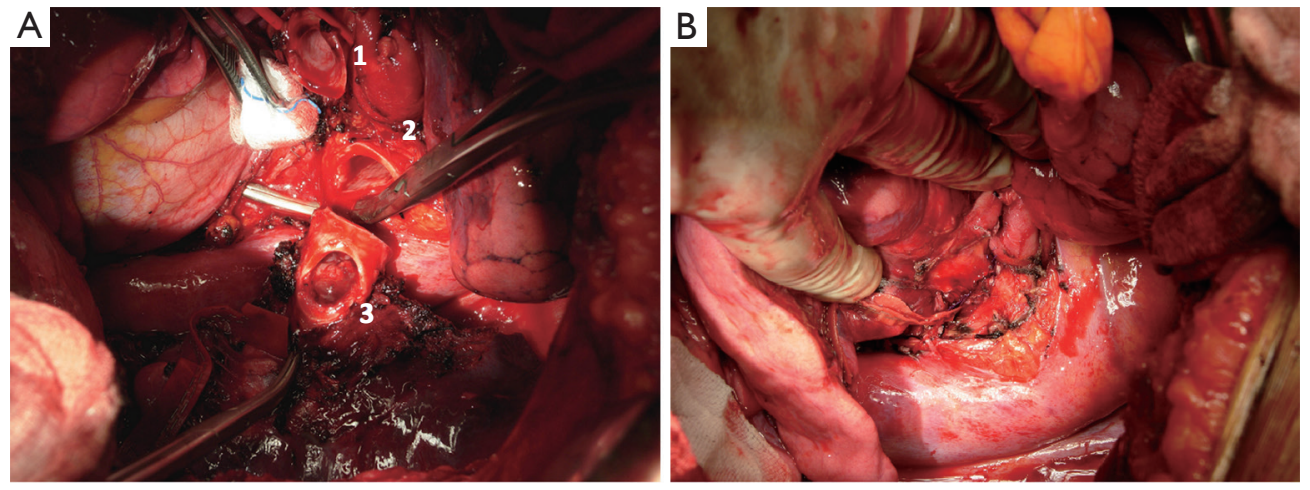

Figure 2 Intraoperative pictures showing left lower sleeve lobectomy (SL) ("Y" sleeve). (A) The bronchi are divided (1, upper lobe bronchial stamp; 2, left main stem bronchial stamp; 3, lower lobe bronchial stamp); (B) completed bronchial anastomosis.

extends up proximal to the upper lobe orifice. The right main stem bronchus is divided at this level just proximal to the right upper lobe take-off and the right upper lobe bronchus is divided at its origin. The upper lobe bronchus is then anastomosed to the main stem bronchus after removal of the middle and lower lobes (the so called "Y" sleeve resection). Due to the reorientation of the upper lobe bronchus after removal of the middle and lower lobes, special care must be taken to avoid torsion of the bronchus at the level of the anastomosis.

For excision of lesions involving the left lower lobe orifice with extension into the main stem bronchus but sparing the upper lobe orifice, a lower lobectomy with sleeve resection of the left main bronchus can be performed. After completion of the bronchial dissection, umbilical tape is passed around the left main stem and left upper lobe bronchus. The left upper lobe bronchus is divided at its origin. Next, the main stem bronchus is divided proximal to the bifurcation and well beyond the extent of the tumor (Figure 2A). Once the margins are confirmed microscopically to be negative by frozen-section analysis, anastomotic reconstruction is performed according to the previously described technique (Figure $2 B$ ). The lingular bronchus may arise proximally to the section line, and care must be taken when dividing the upper lobe bronchus to ensure that it remains intact.

\section{Middle lobe sleeve resection}

Middle lobe sleeve resection is performed infrequently. The bronchus to the middle lobe lies immediately posterior to the middle lobe vein. The bronchus is followed back to its origin. A right-angled clamp is placed around the bronchus intermedius, and this is divided proximally to the middle lobe orifice. The division is slightly angled. The distal division is also angled to preserve the orifice to the superior segment of the lower lobe. The PA lies directly posterior and slightly superior to the bronchus. Therefore, care must be taken to avoid PA injury when dividing the bronchus (Figure $3 A$ ). The arterial branch to the middle lobe is ligated and divided. After confirmation of negative margins, the airway anastomosis is performed according to the previously described technique (Figure 3B).

\section{PA reconstruction}

\section{Indication}

Primary lung tumors and metastatic N1 lymph nodes with extracapsular extension can infiltrate the PA and involve the right the left main branches to different extents (30).

Moreover, residual tumor or scarring tissue after induction therapy can involve the PA and require a sleeve resection.

In cases of limited, marginal infiltration of the arterial wall, a simple tangential resection with direct suture can be sufficient to achieve a radical exeresis. This technique is generally regarded as a variation of a standard resection and therefore is not considered in this article. In larger defects up to $30 \%$ or $40 \%$ of the vessel circumference the reconstruction can be performed by a patch (of biological or synthetic material), avoiding a circumferential resection. More extended infiltration requires a sleeve resection and reconstruction by end-to-end anastomosis, or by the interposition of a prosthetic conduit. However, extended infiltration of the PA, such as a left upper lobe tumor infiltrating the concave surface of the PA from its origin down 

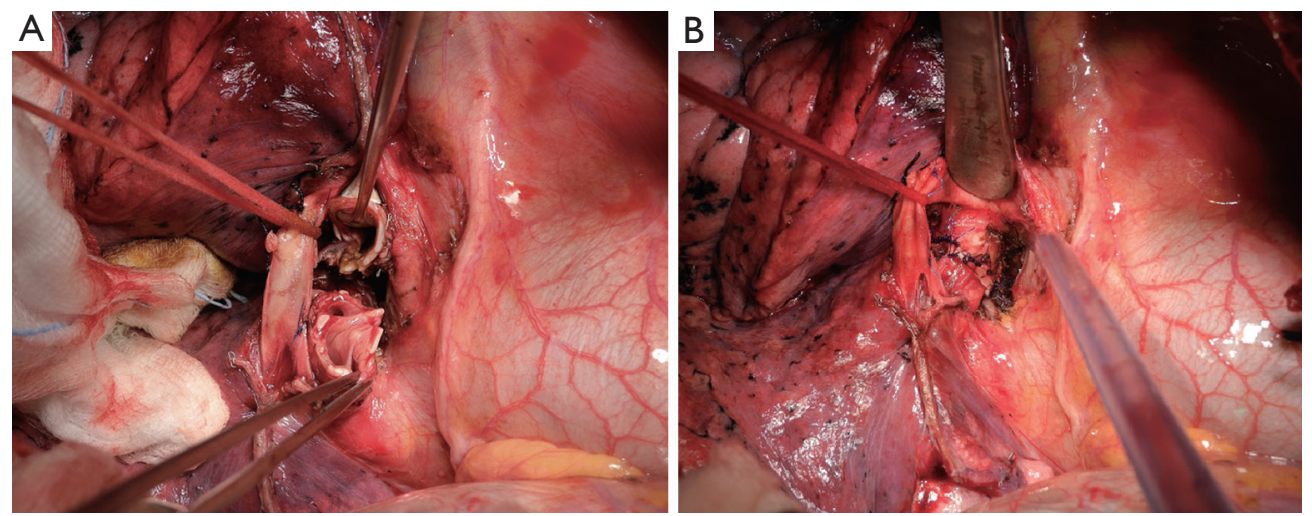

Figure 3 Middle lobe sleeve resection. Intraoperative view. (A) Middle lobe has been removed. The bronchial stamps (the bronchus to the lower lobe and the bronchus intermedius) are visible before anastomotic reconstruction. The pulmonary artery (PA) is encircled with a surgical umbilical tape and retracted; (B) completed bronchial anastomosis.

to the anterobasal artery or, on the right side, posterolateral infiltration from the upper division artery to the artery for the superior segment of the lower lobe, make PN mandatory in order to achieve complete tumor resection.

\section{Operative technique}

The first step of the operation consists in achieving full control of the proximal portion of the PA. Although division of the superior PV can facilitate the exposure of the $\mathrm{PA}$, transection of this vessel should be postponed until the feasibility of the procedure has been ascertained.

The resection phase begins once the main and distal $\mathrm{PA}$, the bronchus and both PVs have been duly prepared. The superior PV is generally divided first. Clamping of the proximal PA is then performed after systemic heparinization. In the past we used to clamp the inferior PV to obtain backflow control. Actually, we prefer to clamp the PA distally to the tumor infiltration.

The dose of intravenous heparin represents the only intraoperative management modification adopted by the authors over time. We actually prefer to administer 1,5002,000 units (about 25 units $/ \mathrm{kg}$ ) instead of a dose between 3,000 and 5,000 units used in the past. Heparin dose has been reduced to prevent postoperative oozing, especially from the lymphadenectomy sites. We have observed that, with progressive reduction of mean clamping time due to increased experience, this dose proves effective in avoiding the risk of thrombosis. Heparin is not reversed by protamine after decamping, once the vascular reconstruction has been completed.

\section{Partial resection and patch reconstruction}

Patch reconstruction can be used in a variety of conditions ranging from limited infiltration of the origin of segmental arteries to larger resection of the PA involving less than one half of the vessel circumference. If the infiltration is more extended a sleeve resection with end-to-end anastomosis or conduit interposition has to be performed.

Various materials have been proposed and used for patch reconstruction, including synthetic or biological options. One biological option is represented by the venous patches including azygos, saphenous and superior PV patches. Azygos vein patches present adequate characteristics, but are available only on the right side and provide limited amount of tissue. Conversely, superior PV can provide similar characteristics to the vessel to be reconstructed, but superior PV patch can be generally obtained in case of concomitant left upper lobectomy, since anatomical reasons make its availability more difficult on the right side. When the extra-parenchymal portion of PA is free from tumor, the PV can be closed proximally by a thoraco-abdominal (TA) stapler $(30 \mathrm{~mm})$ and is ligated more distally at the extralobar origin of its branches. Then it is sectioned proximally and distally between sutures, so that a $1-2 \mathrm{~cm}$ conduit is obtained; a patch adequate for PA reconstruction can be easily obtained trimming the PV conduit.

Among the biological materials, the authors recommend the use of the autologous, bovine or porcine pericardium. In particular, they prefer the autologous pericardium because it has a number of advantages: it shows adequate thickness and resistance, it is cost-free and available on both sides of the chest, and it has superior biocompatibility if compared with 


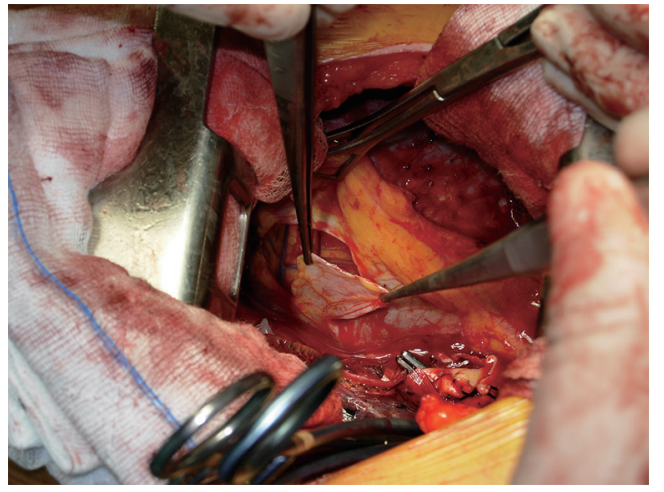

Figure 4 The introperative picture shows the harvesting of the autologous pericardium performed anteriorly to the left phrenic nerve for pulmonary artery $(\mathrm{PA})$ patch reconstruction.

the bovine pericardium. Moreover, it provides an amount of tissue that is sufficient also for large defect repair, and does not require a separate surgical procedure for its harvesting. However, fresh autologous pericardium has some technical limits, since it has a tendency to shrink and curl making more difficult the adaptation and suturing of the patch to the vascular wall.

Bovine pericardium displays limited elasticity and exhibits even and stiff edges that considerably reduce the pitfall of harvesting, trimming and suturing the autologous pericardium.

In order to improve technical features of the autologous pericardium, over the last decade, the authors have devised and employed an intraoperative method of fixation of the patch by a glutaraldehyde-buffered solution (19). The glutaraldehyde preservation of the pericardium minimizes its tendency to retract and curl, thus allowing an easier vascular reconstruction, and reducing the risk of bleeding from the patch suture related to the elastic recoil of the autologous pericardium. Harvesting of the autologous pericardium is performed anteriorly to the phrenic nerve, leaving open the pericardial defect (Figure 4).

The patch is appropriately trimmed and secured to the arterial wall by two stay sutures at the opposite sides of the vascular defect. Suturing is done with $5-0$ or 6-0 monofilament non-absorbable material proceeding from top-to-bottom "artery first", and then continuing from bottom-to-top "patch first", while the assistant grasps and stretches the patch. The inferior stay suture is not tied: it is used only to keep the patch in place and is removed when the suture line reaches its level.

In patients requiring also a bronchial anastomosis, the PA patch reconstruction is generally performed first, in order to reduce clamping time.

Check of the suture line after residual lung re-expansion is mandatory, especially if fresh autologous pericardium is used for reconstruction, since retraction of the patch margins may result in bleeding sites when tension changes are applied on the PA axis.

\section{Sleeve resection and reconstruction by end-to-end anastomosis}

In patients that require a combined bronchovascular sleeve PA reconstruction is usually performed after completion of the bronchial anastomosis to minimize the manipulation of the vessel.

When transecting the artery, both proximally and distally, regular and even margins are desirable, even at the cost of some loss of tissue. This allows proper placement of the stitches and yields an even inside lumen. In addition, regular suture borders facilitate the correction of the large caliber discrepancy that usually occurs. In addition, the exposure of the bronchial stumps is optimal when the artery is divided. If the vascular and bronchial procedures are done simultaneously, the bronchial axis is shortened, and the PA stumps are opposable with acceptable tension. On completion of the bronchial anastomosis, the distance between the two arterial ends will be markedly decreased, and it can be further reduced by elevating the lower lobe while suturing. Restoration of blood flow and removal of the proximal clamp relieves any residual tension. If the distance between the arterial stumps is deemed excessive, the interposition of a prosthetic conduit is indicated. The anastomosis is performed with running $5-0$ or 6-0 monofilament non-absorbable material. Additionally, the sutures are placed very carefully to avoid stenosis. End-to-end anastomosis can be technically difficult due to unexpected traction between the stumps and caliber discrepancy.

\section{Long-segment PA reconstruction}

In case of isolated extended PA defects more than $2.5 \mathrm{~cm}$ in length it could be indicated a reconstruction by a wide patch or by a prosthetic conduit.

Wide patch reconstruction is indicated for longitudinal defects on one aspect of the PA when the opposite side of the circumference of the vessel is free from tumor (Figure 5). If this is not the case, an interposition of a prosthetic conduit is required. Patch reconstruction technique has been previously described. In the following paragraph we 
are describing the conduit reconstruction.

Sleeve resection and reconstruction by a prosthetic conduit. In some patients after sleeve resection of the PA an excessive distance between the two vascular stumps may result. This condition could produce an high tension on the anastomosis. Such technical situation may occur, usually on the left side, in those cases requiring resection of a long segment of the PA without associated bronchial sleeve resection, because the lobar bronchus is not involved. In these cases the vascular reconstruction cannot be performed by a direct end-to-end anastomosis and a prosthetic conduit interposition is required.

Although the need for a vascular conduit is not a frequent

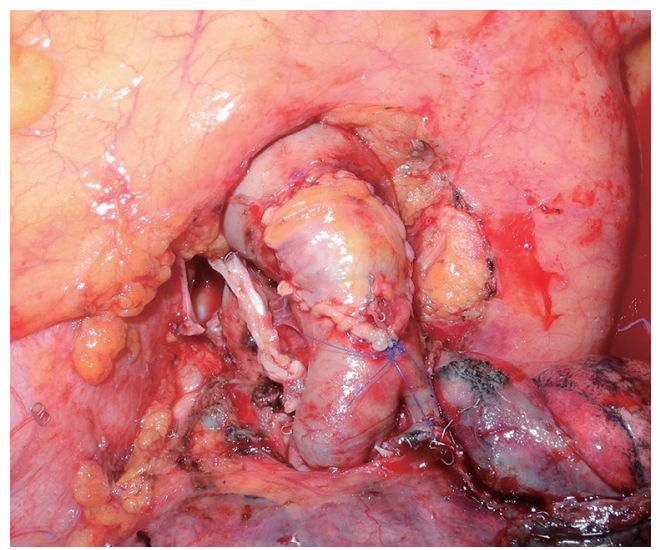

Figure 5 Intraoperative picture illustrating completed longsegment left pulmonary artery (PA) reconstruction by a wide patch of superior pulmonary vein (PV). condition, various materials and different techniques have been proposed for such reconstructive procedure.

Biological materials are generally preferred because of higher biocompatibility and lower risk of thrombosis. The authors have reported the successful use of the autologous and the bovine pericardium (31). More recently, the current authors have introduced the use of porcine pericardium. Intraoperatively, the pericardial leaflet is trimmed to a rectangular shape and wrapped around a chest tube or a syringe of appropriate diameter and sutured longitudinally. In our initial experience this suture was performed manually with a 6-0 monofilament non-absorbable material. More recently we have described a technical alternative with a mechanical suture using a linear stapler for the conduit construction (Figure 6). The creation of a 1-2 cm conduit is so accomplished. When the autologous pericardium is employed the epicardial surface is oriented inside the conduit lumen.

A very interesting alternative for conduit reconstruction is represented by the superior PV of the resected upper lobe when the extra-parenchymal portion of this vessel is free from tumor $(20,32)$. The technique used in order to obtain the $\mathrm{PV}$ conduit is the same described in the previous paragraph for $\mathrm{PV}$ patch tailoring.

The venous conduit is an ideal substitute for PA replacement since it has adequate thickness and structural similarity with the arterial wall. It is advisable to tailor the length of the biological conduit on the basis of the resected arterial segment, because the elasticity of the two tissues is comparable.
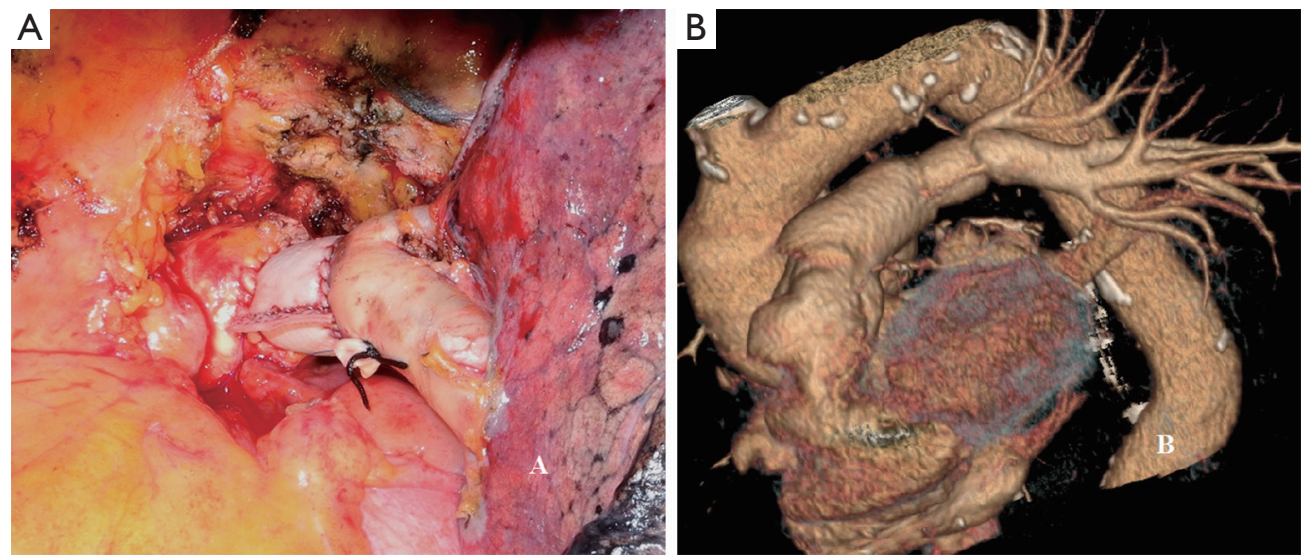

Figure 6 Long-segment pulmonary artery (PA) reconstruction by porcine pericardium conduit. (A) Completed reconstruction of the PA; intraoperative view; (B) computed tomography (CT) with volume rendering showing the complete patency of the reconstructed PA 1 year after surgery; mechanical stapler line between the proximal and distal anastomoses is visible. 
The proximal anastomosis is performed first with running 5-0 monofilament suture. The distal anastomosis is then performed with the same technique, after the conduit length has been checked.

Care must be taken to avoid lengthening of the reconstructed PA, which may cause kinking of the vessel, impaired blood flow and therefore thrombus formation.

For the final success of the reconstruction, it is fundamental to avoid tension on the anastomosis. Tension release can be improved by sectioning the inferior pulmonary ligament and, on the right side, by opening the pericardium around the inferior PV.

\section{Technical and perioperative issues}

The good final outcome in every parenchymal sparing reconstructive procedure is principally the result of a meticulous surgical technique. However, there are some critical and controversial aspects concerning intraoperative and perioperative management of a bronchial sleeve resection and/or PA reconstruction that are of a different nature and may determine the outcome. In order to take stock of what we have learned from these lung-sparing operations over time, we have analyzed some of the most important aspects independently in this section.

An aspect that still remains controversial is the use of postoperative steroids in patients undergoing bronchial resection. We believe that the postoperative use of low dose steroids is favorable because it reduces secretion retention and atelectasis, facilitates parenchymal reexpansion, and minimizes the risk of dehiscence and granuloma formation. Aerosolized steroids are also part of postoperative treatment.

When considering the role of bronchoscopy in these complex lung-sparing operations, it is important that an endoscopic examination is performed by one of the operating surgeons in candidates for a sleeve resection. This is advantageous at the time of the operation, when the bronchi are incised and divided. It is also useful to have precise knowledge of the preoperative and intraoperative appearance of the airway to detect a stiffness of the bronchial wall that may indicate peribronchial tumor infiltration. This is crucial in areas where the bronchus is known to be adjacent to the PA, which consequently might be involved.

Routine bronchoscopies are performed at the end of the surgical procedure, before discharge from the hospital, and after 1 and 6 months.
Bronchoarterial fistula can be effectively prevented by interposing a viable tissue flap between the two structures. The use of mediastinal fat pad, pericardial flap, or pleural flap has been reported $(27,29)$. However, an intercostal muscle flap is preferable because of its excellent vascularization provided by the intercostal artery (28).

The preparation of the flap is performed before opening the chest, and the rib retractor is not inserted until the procedure is completed to avoid crushing the intercostal vessels. The periosteum of the fifth rib is incised and then separated from the bone in continuity with the underlying intercostal muscle. Care must be taken to preserve the muscular insertion to the periosteum to avoid injuring the intercostal neurovascular bundle. The intercostal muscle is then incised in the vicinity of the underlying sixth rib and the anterior insertion of the flap is divided. The pedicle is ligated at its anterior extremity. When the bronchial anastomosis is completed, a large right-angle clamp is slid between the PA and the bronchus, and the suture at the extremity of the flap is slid backward around the bronchial anastomosis and between the bronchus and the PA. The flap is then twisted until its pleural side is in contact with the bronchial anastomosis and the pleura are secured to the bronchus by interrupted absorbable 4-0 sutures.

A main concern in bronchial and vascular reconstructive procedures is avoiding tension on the anastomosis.

During a PA patch reconstruction procedure, if some degree of tension exists, it is safer to complete the posterior portion of the suture and subsequently parachute the two stumps together while lifting the lower lobe. When a patch reconstruction is associated with a bronchial sleeve, the bronchial axis is shortened, and the length of the artery remains stationary. The PA may tend to kink and fold over on itself. The repositioning of the PA caused by the re-expansion of the lower lobe may increase this risk further. Impairment of blood flow may ensue, and thrombosis may occur. Under these circumstances, it is better to cut away the distorted segment and proceed to an end-to-end anastomosis.

During an end-to-end PA anastomosis reconstruction, dividing the pulmonary ligament or, more often on the right side, opening the pericardium around the PV can be useful to obtain a tension-free suture.

The main pitfall in the use of a prosthetic conduit for PA reconstruction is sizing its length. Application of the proposed technical issues will prevent this problem. The current authors believe that the autologous PV conduit is preferable for PA reconstruction because it is very easy to use and because other materials might increase the 
risk of thrombosis. Autologous vein conduit is fresh and unpreserved, cost-free, and biocompatible. Conversely, bovine and porcine pericardial tissue are less cost effective and less biocompatible.

Non-invasive radiologic techniques such as magnetic resonance angiography (MRA) or computed tomography (CT) scan with contrast medium and three-dimensional (3D) volume rendering provide outstanding imaging of the PA and may be very useful in demonstrating patency problems even in the immediate postoperative period. In the absence of clinical symptoms, pulmonary angiograms in addition to perfusion lung scans are redundant. In the long term, CT with contrast medium has proved to be a handy, noninvasive diagnostic tool that is useful to evaluate both PA patency and distal PA branching, as well as the overall oncologic status of the patient.

When PA reconstruction is required, appropriate anticoagulation management is crucial. Initial reports (33) did not clarify this important aspect. Historically, systemic anticoagulation was initiated during operation (3,000 to 5,000 U heparin sodium) (34) and maintained by subcutaneous injection of heparin $(15,000 \mathrm{U} /$ day $)$ for 7 to 10 days. We now believe that intravenous injection of $1,500 \mathrm{U}$ heparin sodium during the resection phase without reversal by protamine sulphate at the end of the procedure $(27,32)$, as well as 6,000 U/day low weight molecular heparin administered subcutaneously for 7 days after surgery is sufficient.

\section{Induction therapy}

When considering patients with locally advanced nonsmall cell lung cancer, induction chemotherapy or chemoradiotherapy has become a standardized indication especially in the presence of N2 disease. However, although the beneficial prognostic effects of neoadjuvant therapy have largely been proved, concern about an increased risk of complications when complex reconstructive procedures are performed after oncologic treatment, has limited the diffusion of such operations within multimodality treatment options. Additional risks may be related to the increased difficulty in surgical dissection caused by diffuse fibrotic reaction, and to the potential healing impairment of the reconstructed bronchus caused by tissue damage and compromised vascularization.

After induction treatment, the dissection of the pulmonary hilum and mediastinum can be difficult and hazardous because the bronchial and vascular structures may be embedded in the desmoplastic reaction and scarring tissue produced by the chemotherapy and radiotherapy. Technical expertise and mature surgical judgment are needed, because it is generally in this step of the operation that the tumor can be judged amenable to a sleeve resection or a PN, or considered unresectable. Shrinkage of tumor and fibrosclerotic reaction produced as a consequence of induction therapy usually increase the technical complexity of surgical dissection and may pose doubt in the identification of viable tumor at this site. Frozen-section histology should therefore be performed on all suspicious tissue. However, after chemotherapy or chemoradiotherapy, sleeve resection with reconstructive procedures may also be indicated when indissociable fibrotic tissue with no residual tumor is embedded in the bronchus and/or the PA. In some situations both the upper lobe bronchus and the PA can be encased in fibrotic tissue even without tumor cells at frozen-section analysis. Because lobectomy is technically impossible and $\mathrm{PN}$ is the alternative, we think that this is a good indication for a combined bronchovascular reconstruction. In their initial experience the current authors first reported in 1997 the possibility of performing bronchial and arterial sleeve resection after induction chemotherapy with no mortality, no bronchial and vascular complications, and no local recurrence (L-recurrence) in the airway. The overall perioperative morbidity rate in a series of 27 patients was similar to that reported in patients undergoing post induction standard resection in the same period. In addition, 1- and 4-year survival rates $(78 \%$ and $39 \%)$ did not show significant differences from those reported in the standard resection series $(65 \%$ and $36 \%)(35,36)$. These results have subsequently been reproduced and further developed by other investigators worldwide, but available data remain limited, with the largest published series including fewer than 50 patients (37).

In 2013 the authors published the long-term results of their experience comparing SL with PN after induction chemotherapy (17). A total of 39 patients undergoing bronchial and/or vascular reconstruction associated with lobectomy were analyzed and compared with 39 patients undergoing $\mathrm{PN}$ over a 14-year period. Postoperative complications occurred in $28.2 \%$ of patients receiving bronchovascular reconstruction and in $33.3 \%$ of the PN group, without statistically significant difference between the two surgical options. Complications related to the reconstructive procedure occurred in one patient: a late stenosis of the bronchial anastomosis was observed and it 
was successfully treated by laser and stenting. Postoperative mortality rate in the PN group was $2.6 \%$, while there was no mortality in the SL group. Difference in postoperative mortality was not significant $(\mathrm{P}=0.3)$.

The tumor recurrence rate was $20.5 \%$ in the SL group (loco-regional in 2 patients, distant in 6) and $30.8 \%$ in the PN group (loco-regional in 1 patient, distant in 11), but this difference was not significant. In particular there was no significant difference between the two groups if considering loco-regional recurrence rate only (Table 1).

\section{Results of surgery}

When considering experiences reported in the literature during the last 20 years, the analysis of long-term survival according to stage and nodal status shows that SL results in higher survival rates for stages I and II. The survival advantage in stage III patients appears to be limited $(7,11,14)$, and the role of parenchymal sparing operations in patients with $\mathrm{N} 2$ disease still remains undefined $(8,38)$.

Nagayasu and colleagues (18) comparing long-term results of bronchovascular reconstructions performed in two different time periods over 30 years' experience, reported results dramatically influenced by the high rate of $\mathrm{pN} 2$ and even $\mathrm{pN} 3$ patients treated during the first period, thus indicating the importance of a correct surgical indication when performing reconstructive procedures for lung cancer.

It has been demonstrated $(34,39)$ that the survival of patients undergoing PA reconstruction is comparable, stageby-stage, to that reported in the major reviews on lung cancer surgery and sleeve resection in the literature. The impact of nodal status on survival is also comparable to that reported for bronchial sleeve and standard resection. Once the decision to resect the disease with intent to cure is taken, $\mathrm{PA}$ reconstruction can also be proposed as an adequate procedure in this setting. Moreover, there is no significant difference between PA reconstruction alone and PA reconstruction associated with bronchial sleeve in terms of postoperative mortality and morbidity (40). One apparently new datum that surfaces from our more recent study (39) and is different from our previous report (34) is that combined bronchovascular reconstructions may offer better survival. This suggests that even this complex lung-sparing operations can be pursued with intent to cure as long as a complete anatomic resection is achieved (11,34,39-43).

However, postoperative morbidity and mortality data reveal overall better results for patients undergoing SL with respect to $\mathrm{PN}$ (Table 1) $(5,6,14,15)$.
An interesting meta-analysis (1) including series published between 1996 and 2006 has compared early and long-term outcome of SL with those of PN. A total of 2,984 patients have been included in this analysis, of which $21 \%$ undergoing SL and 79\% undergoing PN. Two-hundredtwo patients underwent $\mathrm{PA}$ resection and reconstruction in association (164 patients) or not (38 patients) with a bronchial sleeve resection.

Morbidity evaluation from eight studies $(5,6,8-10$, $12,44,45)$ showed a pooled incidence of $31.3 \%$ in the SL group and of $31.6 \%$ in the PN group without statistically significant difference. Similar results were observed limiting the analysis to studies reporting a larger experience (more than 50 patients) of SL. The mean postoperative complication rate reported after PA reconstruction was similar $(32.4 \%)$ to that reported after bronchial SL and PN. Overall postoperative mortality presented a pooled incidence of $3.5 \%$ in the SL group and of $5.7 \%$ in the $\mathrm{PN}$ group, but this difference did not reach statistical significance. However, when considering only studies with larger number (over 50) of SL, mortality rate was significantly lower in the SL group than in the PN group $(5-9,11,12,46)$.

Literature data show that $\mathrm{PN}$ patients appear to experience a higher rate of cardiac complications when morbidity is evaluated according to the type of complication, while SL patients show increased pulmonary and airway complications incidence $(5,6,8,10,38)$.

Overall 5-year survival rate from ten studies (6-12,43-45). was $50.3 \%$ after SL and $30.6 \%$ after PN, showing a statistically significant difference. The median overall survival was 60 months for the SL group and 28 months for the PN group. This result may have been partially influenced by the higher rate of stage III patients included in the PN group in most studies. However, when considering survival according to pathological $\mathrm{N}$ status, pooled 1-year and 5-year survival rate of patients with N0 or N1 disease are significantly higher after SL.

The pooled loco-regional recurrence rate from studies considered in this meta-analysis resulted $16.1 \%$ in the SL group and $27.8 \%$ in the PN group, but this difference did not reach statistical significance. Otherwise, a significantly lower incidence of L-recurrence in favour of SL (SL, 14.5\% $v s$. PN, 28.7\%) was reported in the studies with larger number of sleeve procedures $(6,7,9,12)$.

The preservation of lung parenchyma has been indicated by some authors as the possible cause of a theoretical increased risk for loco-regional recurrence 
after SL. However although in some experiences (38) a higher L-recurrence rate is reported for sleeve resection with advanced nodal status (N2), the few studies $(10,38)$ analyzing risk factors for recurrence, show that the tumor stage and the nodal status are the only negative predictive factors, rather than the type of operation performed.

Literature data also indicate that lung parenchyma sparing improves postoperative quality of life determining a greater cardio-pulmonary reserve, less pulmonary edema and less right ventricular dysfunction due to a lower pulmonary vascular resistance (47). In an interesting paper from Ferguson the Quality Adjusted Years Quoted was 4.37 after SL and 2.48 after PN (29). Another paper from Melloul has retrospectively analysed postoperative FEV1 reporting significantly higher values for patients undergoing SL (13). In a prospective study by Martin-Ucar the reported mean FEV1 loss after parenchymal sparing operations was considerably less than after $\mathrm{PN}$, indicating a strongly significant prognostic advantage for patients undergoing SL.

\section{Conclusions}

It is common knowledge that the results of bronchial and PA sleeve resection improved with increasing experience. When these operations are performed with correct indications, accurate and complete preoperative staging in adjunction with careful surgical technique and perioperative management, they may provide functional advantages of standard lobectomy associated with oncological radicality of PN, even after induction therapy. Results of our and other published experiences support these operations also in patients without functional impairment.

\section{Acknowledgements}

We wish to thank Dr. Marta Silvi for data management and editorial work.

\section{Footnote}

Conflicts of Interest: The authors have no conflicts of interest to declare.

\section{References}

1. Ma Z, Dong A, Fan J, et al. Does sleeve lobectomy concomitant with or without pulmonary artery reconstruction (double sleeve) have favorable results for non-small cell lung cancer compared with pneumonectomy? A meta-analysis. Eur J Cardiothorac Surg 2007;32:20-8.

2. Shi $W$, Zhang $W$, Sun $H$, et al. Sleeve lobectomy versus pneumonectomy for non-small cell lung cancer: a metaanalysis. World J Surg Oncol 2012;10:265.

3. Maurizi G, Ibrahim M, Andreetti C, et al. Long-term results after resection of bronchial carcinoid tumour: evaluation of survival and prognostic factors. Interact Cardiovasc Thorac Surg 2014;19:239-44.

4. Ibrahim M, Maurizi G, Venuta F, et al. Reconstruction of the bronchus and pulmonary artery. Thorac Surg Clin 2013;23:337-47.

5. Gaissert HA, Mathisen DJ, Moncure AC, et al. Survival and function after sleeve lobectomy for lung cancer. J Thorac Cardiovasc Surg 1996;111:948-53.

6. Okada M, Yamagishi H, Satake S, et al. Survival related to lymph node involvement in lung cancer after sleeve lobectomy compared with pneumonectomy. J Thorac Cardiovasc Surg 2000;119:814-9.

7. Deslauriers J, Grégoire J, Jacques LF, et al. Sleeve lobectomy versus pneumonectomy for lung cancer: a comparative analysis of survival and sites or recurrences. Ann Thorac Surg 2004;77:1152-6; discussion 1156.

8. Ludwig C, Stoelben E, Olschewski M, et al. Comparison of morbidity, 30-day mortality, and long-term survival after pneumonectomy and sleeve lobectomy for non-small cell lung carcinoma. Ann Thorac Surg 2005;79:968-73.

9. Bagan P, Berna P, Pereira JC, et al. Sleeve lobectomy versus pneumonectomy: tumor characteristics and comparative analysis of feasibility and results. Ann Thorac Surg 2005;80:2046-50.

10. Kim YT, Kang CH, Sung SW, et al. Local control of disease related to lymph node involvement in non-small cell lung cancer after sleeve lobectomy compared with pneumonectomy. Ann Thorac Surg 2005;79:1153-61; discussion 1153-61.

11. Lausberg HF, Graeter TP, Tscholl D, et al. Bronchovascular versus bronchial sleeve resection for central lung tumors. Ann Thorac Surg 2005;79:1147-52; discussion 1147-52.

12. Takeda S, Maeda H, Koma M, et al. Comparison of surgical results after pneumonectomy and sleeve lobectomy for non-small cell lung cancer: trends over time and 20-year institutional experience. Eur J Cardiothorac Surg 2006;29:276-80.

13. Melloul E, Egger B, Krueger T, et al. Mortality, complications and loss of pulmonary function after 
pneumonectomy vs. sleeve lobectomy in patients younger and older than 70 years. Interact Cardiovasc Thorac Surg 2008;7:986-9.

14. Parissis H, Leotsinidis M, Hughes A, et al. Comparative analysis and outcomes of sleeve resection versus pneumonectomy. Asian Cardiovasc Thorac Ann 2009;17:175-82.

15. Park JS, Yang HC, Kim HK, et al. Sleeve lobectomy as an alternative procedure to pneumonectomy for non-small cell lung cancer. J Thorac Oncol 2010;5:517-20.

16. Gómez-Caro A, Garcia S, Reguart N, et al. Determining the appropriate sleeve lobectomy versus pneumonectomy ratio in central non-small cell lung cancer patients: an audit of an aggressive policy of pneumonectomy avoidance. Eur J Cardiothorac Surg 2011;39:352-9.

17. Maurizi G, D'Andrilli A, Anile M, et al. Sleeve lobectomy compared with pneumonectomy after induction therapy for non-small-cell lung cancer. J Thorac Oncol 2013;8:637-43.

18. Nagayasu T, Yamasaki N, Tsuchiya T, et al. The evolution of bronchoplasty and broncho-angioplasty as treatments for lung cancer: evaluation of 30 years of data from a single institution. Eur J Cardiothorac Surg 2016;49:300-6.

19. D'Andrilli A, Ibrahim M, Venuta F, et al. Glutaraldehyde preserved autologous pericardium for patch reconstruction of the pulmonary artery and superior vena cava. Ann Thorac Surg 2005;80:357-8.

20. D'Andrilli A, Maurizi G, Andreetti C, et al. Pulmonary artery reconstruction with pulmonary vein conduit for lung cancer: medium-term results. Ann Thorac Surg 2014;98:990-5.

21. Thomas CP. Conservative resection of the bronchial tree. J R Coll Surg Edinb 1956;1:169-86.

22. Allison PR. Course of thoracic surgery in Groningen. Ann R Coll Surg 1954;25:20-2.

23. Mahtabifard A, Fuller CB, McKenna RJ Jr. Video-assisted thoracic surgery sleeve lobectomy: a case series. Ann Thorac Surg 2008;85:S729-32.

24. Gonzalez-Rivas D, Fernandez R, Fieira E, et al. Uniportal video-assisted thoracoscopic bronchial sleeve lobectomy: first report. J Thorac Cardiovasc Surg 2013;145:1676-7.

25. Rendina EA, De Giacomo T, Venuta F, et al. Lung conservation techniques: bronchial sleeve resection and reconstruction of the pulmonary artery. Semin Surg Oncol 2000;18:165-72.

26. Rendina EA, Venuta F, Ciriaco P, et al. Bronchovascular sleeve resection. Technique, perioperative management, prevention, and treatment of complications. J Thorac
Cardiovasc Surg 1993;106:73-9.

27. Yildizeli B, Fadel E, Mussot S, et al. Morbidity, mortality, and long-term survival after sleeve lobectomy for non-small cell lung cancer. Eur J Cardiothorac Surg 2007;31:95-102.

28. Rendina EA, Venuta F, Ricci P, et al. Protection and revascularization of bronchial anastomoses by the intercostal pedicle flap. J Thorac Cardiovasc Surg 1994;107:1251-4.

29. Tsuchiya R. Bronchoplastic techniques. In: Patterson GA, Deslauriers J, Lerut A, et al., editors. Pearson's Thoracic and Esophageal Surgery. 2nd ed. Philadelphia, PA: Churchill Livingstone, 2002:1005.

30. Ricci C, Rendina EA, Venuta F, et al. Reconstruction of the pulmonary artery in patients with lung cancer. Ann Thorac Surg 1994;57:627-32; discussion 632-3.

31. Rendina EA, Venuta F, De Giacomo T, et al. Reconstruction of the pulmonary artery by a conduit of autologous pericardium. J Thorac Cardiovasc Surg 1995;110:867-8.

32. Cerezo F, Cano JR, Espinosa D, et al. New technique for pulmonary artery reconstruction. Eur J Cardiothorac Surg 2009;36:422-3.

33. Vogt-Moykopf I, Fritz T, Meyer G, et al. Bronchoplastic and angioplastic operation in bronchial carcinoma: longterm results of a retrospective analysis from 1973 to 1983 . Int Surg 1986;71:211-20.

34. Rendina EA, Venuta F, De Giacomo T, et al. Sleeve resection and prosthetic reconstruction of the pulmonary artery for lung cancer. Ann Thorac Surg 1999;68:9951001; discussion 1001-2.

35. Rendina EA, Venuta F, De Giacomo T, et al. Safety and efficacy of bronchovascular reconstruction after induction chemotherapy for lung cancer. J Thorac Cardiovasc Surg 1997;114:830-5; discussion 835-7.

36. Erino AR, Venuta F, De Giacomo T, et al. Sleeve resection after induction therapy. Thorac Surg Clin 2004;14:191-7, vi.

37. Bagan P, Berna P, Brian E, et al. Induction chemotherapy before sleeve lobectomy for lung cancer: immediate and long-term results. Ann Thorac Surg 2009;88:1732-5.

38. Fadel E, Yildizeli B, Chapelier AR, et al. Sleeve lobectomy for bronchogenic cancers: factors affecting survival. Ann Thorac Surg 2002;74:851-8; discussion 858-9.

39. Venuta F, Ciccone AM, Anile M, et al. Reconstruction of the pulmonary artery for lung cancer: long-term results. J Thorac Cardiovasc Surg 2009;138:1185-91.

40. Alifano M, Cusumano G, Strano S, et al. Lobectomy with pulmonary artery resection: morbidity, mortality, and long- 
term survival. J Thorac Cardiovasc Surg 2009;137:1400-5.

41. Shrager JB, Lambright ES, McGrath CM, et al.

Lobectomy with tangential pulmonary artery resection without regard to pulmonary function. Ann Thorac Surg 2000;70:234-9.

42. Cerfolio RJ, Bryant AS. Surgical techniques and results for partial or circumferential sleeve resection of the pulmonary artery for patients with non-small cell lung cancer. Ann Thorac Surg 2007;83:1971-6; discussion 1976-7.

43. Nagayasu T, Matsumoto K, Tagawa T, et al. Factors affecting survival after bronchoplasty and bronchoangioplasty for lung cancer: single institutional review of 147 patients. Eur J Cardiothorac Surg 2006;29:585-90.

44. Yoshino I, Yokoyama H, Yano T, et al. Comparison of

Cite this article as: Maurizi G, D'Andrilli A, Venuta F, Rendina EA. Reconstruction of the bronchus and pulmonary artery. J Thorac Dis 2016;8(Suppl 2):S168-S180. doi: 10.3978/ j.issn.2072-1439.2016.02.06 the surgical results of lobectomy with bronchoplasty and pneumonectomy for lung cancer. J Surg Oncol 1997;64:32-5.

45. Ghiribelli C, Voltolini L, Luzzi L, et al. Survival after bronchoplastic lobectomy for non small cell lung cancer compared with pneumonectomy according to nodal status. J Cardiovasc Surg (Torino) 2002;43:103-8.

46. Suen HC, Meyers BF, Guthrie T, et al. Favorable results after sleeve lobectomy or bronchoplasty for bronchial malignancies. Ann Thorac Surg 1999;67:1557-62.

47. Ferguson MK, Lehman AG. Sleeve lobectomy or pneumonectomy: optimal management strategy using decision analysis techniques. Ann Thorac Surg 2003;76:1782-8. 